\title{
Spatially Non-Uniform Trap State Densities in Solution-Processed Hybrid Perovskite Thin Films
}

Sergiu Draguta $^{1}$, Siddharatka Thakur ${ }^{1,2}$, Yurii Morozov ${ }^{1}$, Yuanxing Wang ${ }^{1}$, Joseph S. Manser ${ }^{3,4}$, Prashant V. Kamat ${ }^{1,3,4}$, Masaru Kuno ${ }^{1 *}$

${ }^{1}$ Department of Chemistry and Biochemistry, University of Notre Dame, Notre Dame, Indiana 46556, USA

${ }^{2}$ Nanotechnology Engineering, University of Waterloo, Waterloo, Ontario N2L 3G1, Canada

${ }^{3}$ Department of Chemical and Biomolecular Engineering, University of Notre Dame, Notre Dame, Indiana 46556, USA

${ }^{4}$ Radiation Laboratory, University of Notre Dame, Notre Dame, Indiana 46556, USA

\section{*mkuno@nd.edu}

Deriving $b$ coefficient limits for low and high excitation regimes

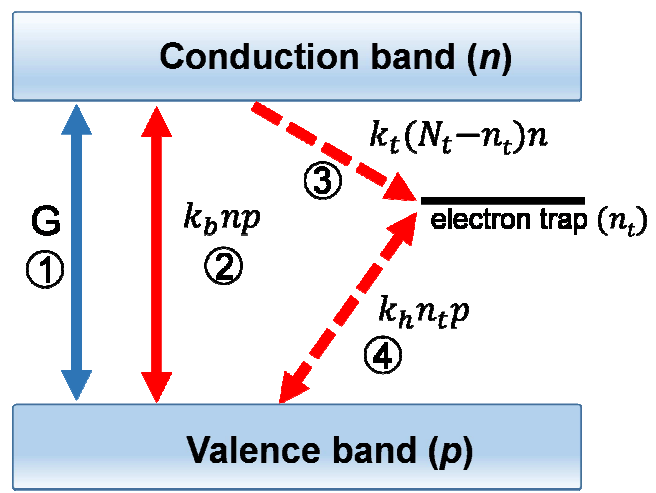

Caption: Schematic representation of the three level system used to model the perovskite emission.

Relevant rate expressions for the different states are:

$$
\begin{aligned}
\frac{d n}{d t} & =G-k_{b} n p-k_{t}\left(N_{t}-n_{t}\right) n \\
\frac{d p}{d t} & =G-k_{b} n p-k_{h} n_{t} p \\
\frac{d n_{t}}{d t} & =k_{t}\left(N_{t}-n_{t}\right) n-k_{h} n_{t} p
\end{aligned}
$$

where, $G$ represents a generation rate $\left(G=\alpha I_{\text {exc }}\right)$ with $\alpha$ an absorption coefficient, $k_{b}$ is a bimolecular radiative recombination rate constant, $k_{t}$ is an electron trapping rate constant, $N_{t}$ represents the total electron trap concentration, and $k_{h}$ is an electron detrapping rate constant.

\section{Low excitation intensities}

At low excitation intensities, trapping dominates. The bimolecular radiative term can therefore be ignored in Equations 1 and 2. This gives 


$$
\begin{aligned}
& \frac{d n}{d t} \approx G-k_{t} N_{t} n \\
& \frac{d p}{d t} \approx G-k_{h} n_{t} p .
\end{aligned}
$$

If the material is intrinsic and all photogenerated electrons are effectively trapped, $n_{t}=p$. Consequently, Equation 5 becomes

$$
\frac{d p}{d t} \approx G-k_{h} p^{2}
$$

At this point, under steady state conditions Equations 4 and 6 yield

and

$$
n=\frac{G}{k_{t} N_{t}}
$$

$$
p=\sqrt{\frac{G}{k_{h}}}
$$

Since $n \propto G$ and $p \propto \sqrt{G}$, the corresponding emission intensity is:

$$
I_{e m} \propto k_{b} n p \propto G^{\frac{3}{2}}
$$

\section{High excitation intensities}

At high excitation intensities, prior to the onset of Auger mediated processes, we assume trap saturation. Consequently, trapping terms can be excluded from Equations 1 and 2. This gives:

$$
\begin{aligned}
& \frac{d n}{d t}=G-k_{b} n p \\
& \frac{d p}{d t}=G-k_{b} n p
\end{aligned}
$$

from where under steady state conditions with $n=p$

and

$$
n=\sqrt{\frac{G}{k_{b}}}
$$

$$
p=\sqrt{\frac{G}{k_{b}}}
$$

Since $n \propto \sqrt{G}$ and $p \propto \sqrt{G}$, the corresponding emission intensity is

$$
I_{e m} \propto k_{b} n p \propto G
$$

\section{Fitting procedure to extract $N_{t}$}

Fitting of the experimental data with the above model was done as follows. First, the following literature estimates for the various rate constants were used ${ }^{1,2}$ 
- $k_{b}=2.6 \times 10^{-10} \mathrm{~cm}^{3} / \mathrm{s}$

- $k_{t}=2.0 \times 10^{-10} \mathrm{~cm}^{3} / \mathrm{s}$

- $k_{h}=8.0 \times 10^{-12} \mathrm{~cm}^{3} / \mathrm{s}$.

Next, a Matlab code was written to fit experimental $I_{e m}$ versus $I_{e x c}$ traces with numerical results of the model. Specifically, intensity-dependent $n$ and $p$ values were found and multiplied by $k_{b}$ to obtain an estimate for $I_{e m}$. $N_{t}$ was used as a global fitting parameter.

To convert $b$ coefficient maps to corresponding $N_{t}$ maps, three $I_{e m}$ versus $I_{e x c}$ traces from a map were first fit using the above described procedure. Associated $N_{t}$ values were then found and were linked to the $b$ coefficient of each trace. A linear (in semilog space) fit to the resulting $N_{t}$ versus $b$ association then provided a calibration curve, which allowed $b$ coefficient maps to be converted to corresponding $N_{t}$ maps.

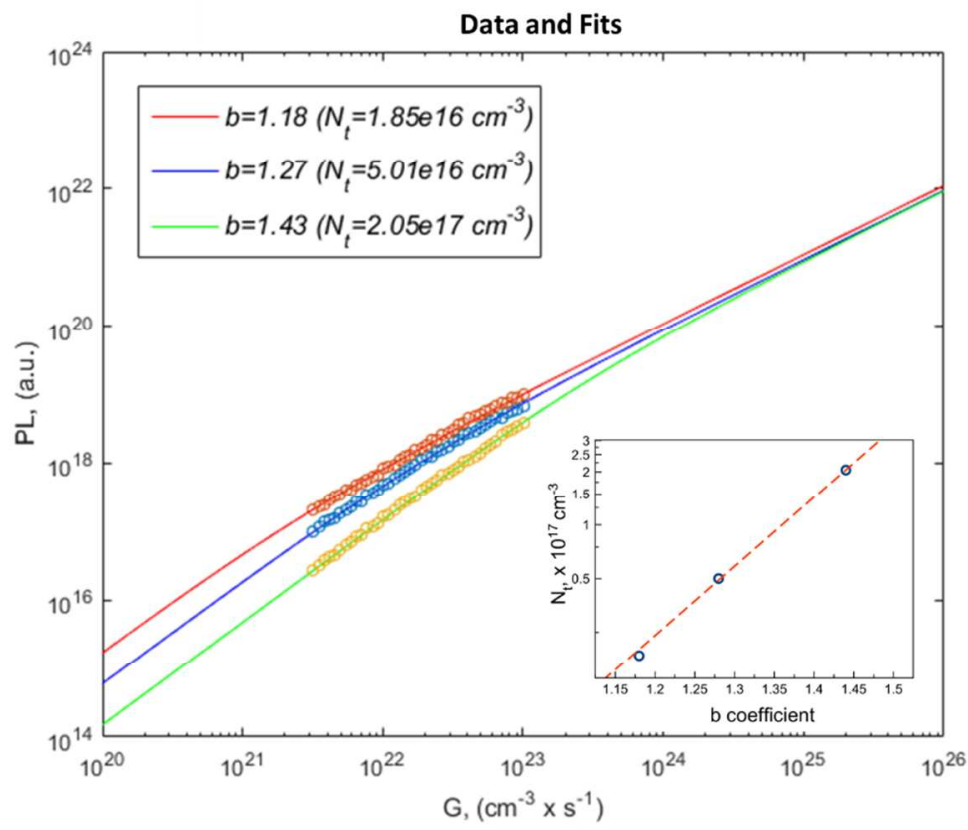

Caption. Fits of $I_{e m}$ versus $I_{e x c}$ traces with different $b$ values. (Inset) generated calibration curve linking $b$ values to $N_{t}$ values.

\section{Derivation of Equation 1}

\section{Act 1}

If we consider the following kinetic picture for carrier recombination in an intrinsic semiconductor 


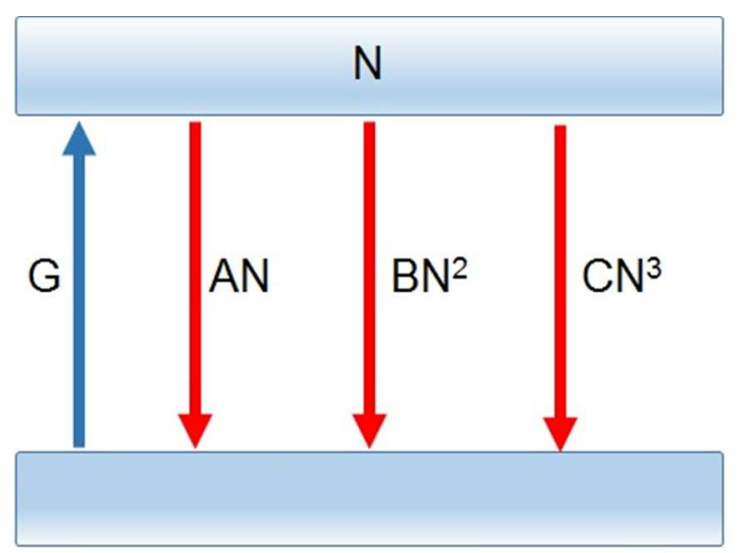

Caption: Kinetic scheme for intensity-dependent QYs

the following rate equation applies

$$
\frac{d N}{d t}=\frac{\alpha I_{e x c}}{h v}-A N-\eta_{e} B N^{2}-C N^{3}
$$

In the expression, $N$ is the photogenerated electron-hole density, $\eta_{e}$ is an output coupling factor that reflects emission losses due internal reflection and other factors, $A$ is an effective first order rate constant accounting for non-radiative, trap-mediated recombination, $B$ is a generic second order bimolecular (radiative) rate constant, and $C$ is a generic third order Auger coefficient.

At steady state, if an absorbed power density is defined as $P_{a b s}=\alpha I_{e x c}$ in Equation 15 one can obtain an equivalent expression for $P_{a b s}$ as follows

$$
P_{a b s}=h v\left[A N+\eta_{e} B N^{2}+C N^{3}\right] .
$$

An analogous emitted light power density can be defined as

$$
P_{e m}=\left(h v_{f}\right)\left(\eta_{e} B N^{2}\right)
$$

where $v_{f}$ is an emission frequency which can, in principle, differ from $v$ (i.e. if a Stokes shift exists). Together with the following expression for the external emission quantum yield

$$
Q Y=\frac{\eta_{e} B N^{2}}{\left[A N+\eta_{e} B N^{2}+C N^{3}\right]}
$$

we can equivalently express the external QY as

$$
\frac{P_{e m}}{P_{a b s}}=\left(\frac{v_{f}}{v}\right) Q Y
$$

If $P_{a b s}$ is related to the incident laser power through $P_{a b s}=\xi P_{e x c}$ where $\xi$ is a constant of proportionality Equation 19 becomes 


$$
\frac{P_{e m}}{P_{a b s}}=\xi\left(\frac{v_{f}}{v}\right) Q Y
$$

where upon defining $\beta=\xi\left(\frac{v_{f}}{v}\right)$, a constant of proportionality, gives

$$
\frac{P_{e m}}{P_{a b s}}=\beta Q Y
$$

We will return to this equation shortly.

\section{Act 2}

As described in the main text and in Equation 18, the emission QY will depend on excitation intensity. This QY function will grow with increasing excitation intensity whereupon it will peak and plateau. The introduction of additional Auger processes at higher intensities will subsequently cause the QY function to fall. Using Equation 18 we can therefore establish the optimal carrier density associated with the maximum QY attainable by taking its first derivative to find the function's extremum (i.e. $\frac{d(Q Y)}{d N}=0$ ). What results is the following expression for the optimal carrier density associated with the peak QY

$$
N_{\text {opt }}=\sqrt{\frac{A}{C}}
$$

The corresponding optimal laser power density $\left(P_{\text {opt }}\right)$ where this occurs is found from Equation 16 where we now assume that the bimolecular radiative rate dominates all non-radiative processes, i.e. $\eta_{e} B N^{2} \gg A N+C N^{3}$. Consequently,

$$
P_{o p t} \approx h v\left[\eta_{e} B N_{o p t}^{2}\right]
$$

and when Equation 22 is used

$$
P_{o p t} \approx h v\left[\eta_{e} \frac{A B}{C}\right]
$$

Finally, the corresponding optimal external quantum efficiency $\left(\eta_{\text {ext }}^{o p t}\right)$ associated with $P_{\text {opt }}$ and $N_{\text {opt }}$ is found from Equation 18 using $N_{\text {opt }}$. This gives

$$
\eta_{\text {ext }}^{o p t}=\frac{\eta_{e} B N_{o p t}^{2}}{\left[A N_{o p t}+\eta_{e} B N_{o p t}^{2}+C N_{o p t}^{3}\right]}
$$

where upon explicit substitution of Equation 18 gives

$$
\eta_{\text {ext }}^{o p t}=\frac{1}{1+\left(\frac{2 \sqrt{A C}}{\eta_{e} B}\right)} \text {. }
$$


When the term in parenthesis is small, a Taylor series approximation (i.e. $\frac{1}{1+x} \approx 1-x$ ) can be invoked to give the desired optimal external QY corresponding to $N_{\text {opt }}$ and $P_{\text {opt }}$

$$
\eta_{\text {ext }}^{o p t} \approx 1-\frac{2 \sqrt{A C}}{\eta_{e} B}
$$

\section{Act 3}

Finally, it is possible to establish an alternate high excitation intensity expression for the external quantum yield in terms of the above optimal values. First, assuming that $\eta_{e} B N^{2} \gg$ $A N+C N^{3}$ and that QYs are generally large we obtain the analogous (general) expression to Equation 23. Namely,

$$
P_{a b s} \approx h v\left[\eta_{e} B N^{2}\right]
$$

Then, together with Equation 23, a normalized incident excitation power can be defined as

$$
\bar{P}=\frac{P_{a b s}}{P_{o p t}}=\frac{N^{2}}{N_{o p t}^{2}}
$$

Under these conditions Equation 18 correspondingly becomes

$$
Q Y=\frac{\eta_{e} B N^{2}}{\eta_{e} B N^{2}+\left[A N+C N^{3}\right]}=\frac{1}{1+\frac{\left[A N+C N^{3}\right]}{\eta_{e} B N^{2}}}
$$

such that a Taylor series approximation yields

$$
Q Y \approx 1-\frac{\left[A N+C N^{3}\right]}{\eta_{e} B N^{2}}
$$

When $N$ is expressed in terms of $\bar{P}$ using Equation 29, we obtain

$$
Q Y(\bar{P})=1-\frac{\left[A \sqrt{\bar{P}} N_{o p t}+C \bar{P}^{\frac{3}{2}} N_{o p t}^{3}\right]}{\eta_{e} B \bar{P} N_{o p t}^{2}}
$$

which reduces to the following expression after some gymnastics

$$
Q Y(\bar{P})=1-\left(\frac{2 \sqrt{A C}}{\eta_{e} B}\right) \frac{[1+\bar{P}]}{2 \sqrt{\bar{P}}}
$$

If we now recall Equation 27, we obtain

$$
Q Y(\bar{P})=1-\left(1-\eta_{\text {ext }}^{o p t}\right) \frac{[1+\bar{P}]}{2 \sqrt{\bar{P}}}
$$


At this point, using Equation 21, we can find an expression relating the experimental data to the QY function

$$
\frac{P_{e m}}{\bar{P}}=\beta Q Y(\bar{P})
$$

whereupon substituting Equation 34 gives our final desired expression

$$
\frac{P_{e m}}{\bar{P}}=\beta\left[1-\left(1-\eta_{\text {ext }}^{\text {opt }}\right) \frac{[1+\bar{P}]}{2 \sqrt{\bar{P}}}\right]
$$

which enables fitting of intensity normalized, experimental $I_{e m}$ data to extract an optimal external quantum yield for samples.

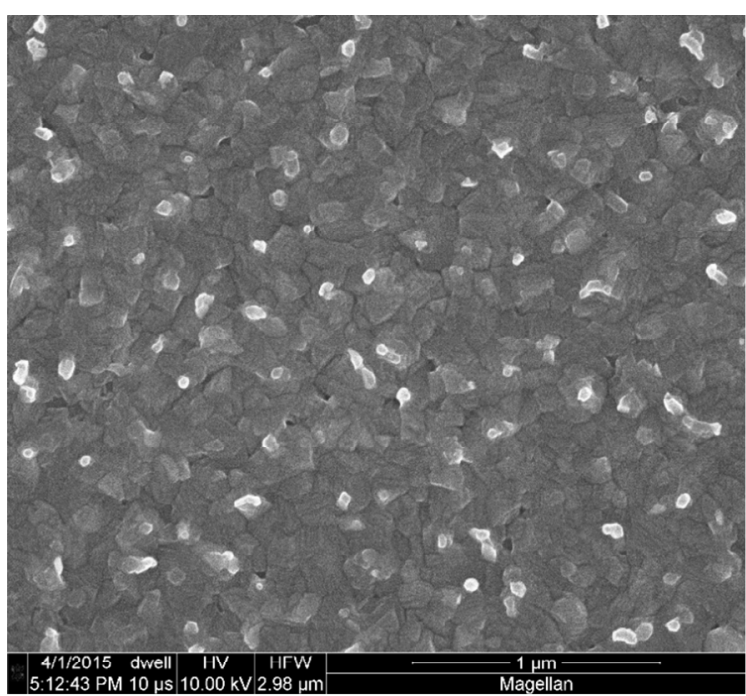

Figure S1. SEM image of a $\mathrm{MAPbI}_{3}$ film. 

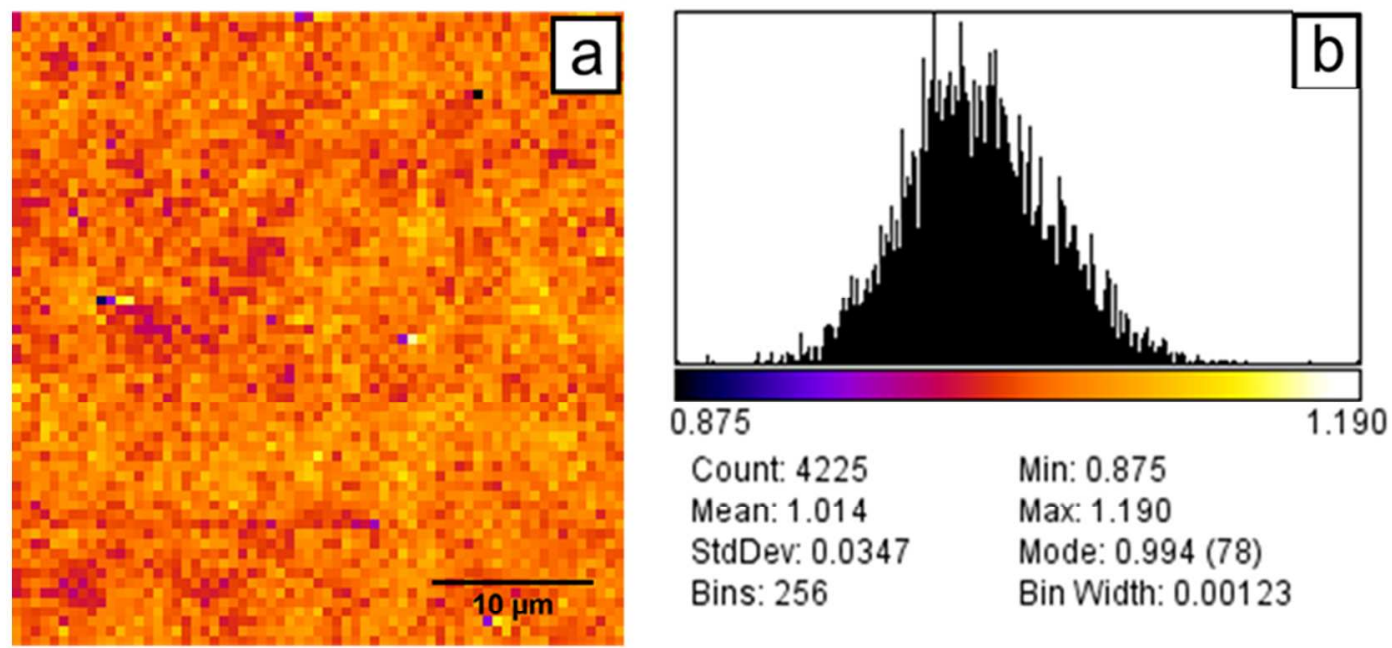

Count: 4225

Mean: 1.014

Min: 0.875

StdDev: 0.0347

Bins: 256

Max: 1.190

Mode: $0.994(78)$

Bin Width: 0.00123

Figure S2. (a) $b$ coefficient map taken at high excitation intensities $\left(I_{\text {exc }}=10^{1}-10^{3} \mathrm{~W} / \mathrm{cm}^{2}\right)$. (b) Histogram showing an average $b$ coefficient of $b=1.01$ along with the experimental distribution about the mean.

\section{Quantum yield map details}

Local QYs are established using the data in the inset of Figure 1a. First, a fit of $I_{e m}$ versus $I_{\text {exc }}$ to Equation 1 yields a maximum QY of $\eta_{\text {ext }}^{o p t}=70 \%$ associated with an optimal excitation intensity of $I_{\text {exc }}^{\max }=30 \mathrm{~W} / \mathrm{cm}^{2}$. Then, the corresponding QY at 1 Sun is determined using this calibration point. We find a 1 Sun QY of QY $=5.3 \%$. Next, given that the thin film $I_{e m}$ versus $I_{\text {exc }}$ (Figure 1a inset) possesses a power law coefficient of $b \approx 1.5$ over the range of $I_{e x c}$ values used in 2D maps $\left(I_{e x c}=10^{-2}\right.$ to $10^{0} \mathrm{~W} / \mathrm{cm}^{2}$ ), points in maps where $b \sim 1.5$ (actual $\mathrm{b}$ coefficients between $b=1.49-1.51$ ) are identified (e.g. Figure 2a, main text) and their integrated emission intensities (e.g. Figure 3a, main text) are compiled in a histogram (see below). The average integrated emission intensity is subsequently associated with the $5.3 \% 1$ Sun QY. Finally, by 
assuming a direct correlation between integrated emission intensities and QYs, all other points in the 2D map are assigned 1 Sun QYs.

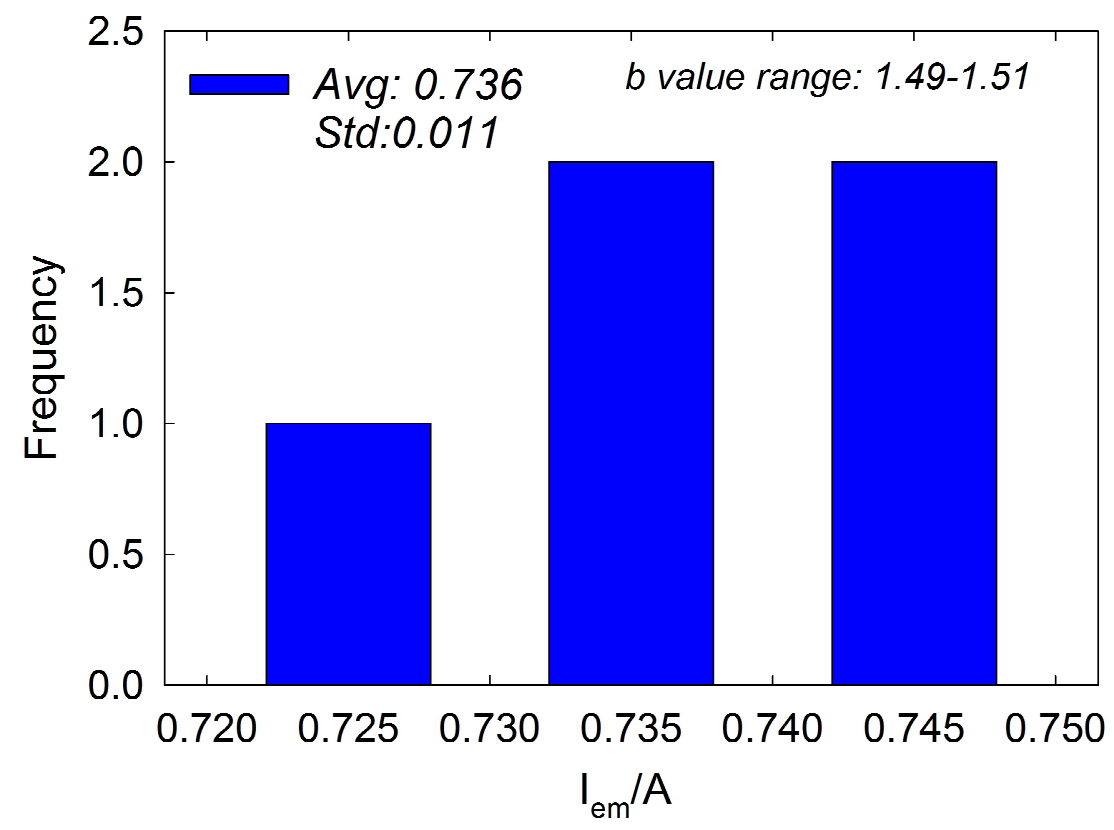

Caption: Distribution of normalized $I_{e m} / A$ for points in the $2 \mathrm{D}$ integrated emission map associated with $b$ coefficients in the range $b=1.49-1.51$. 


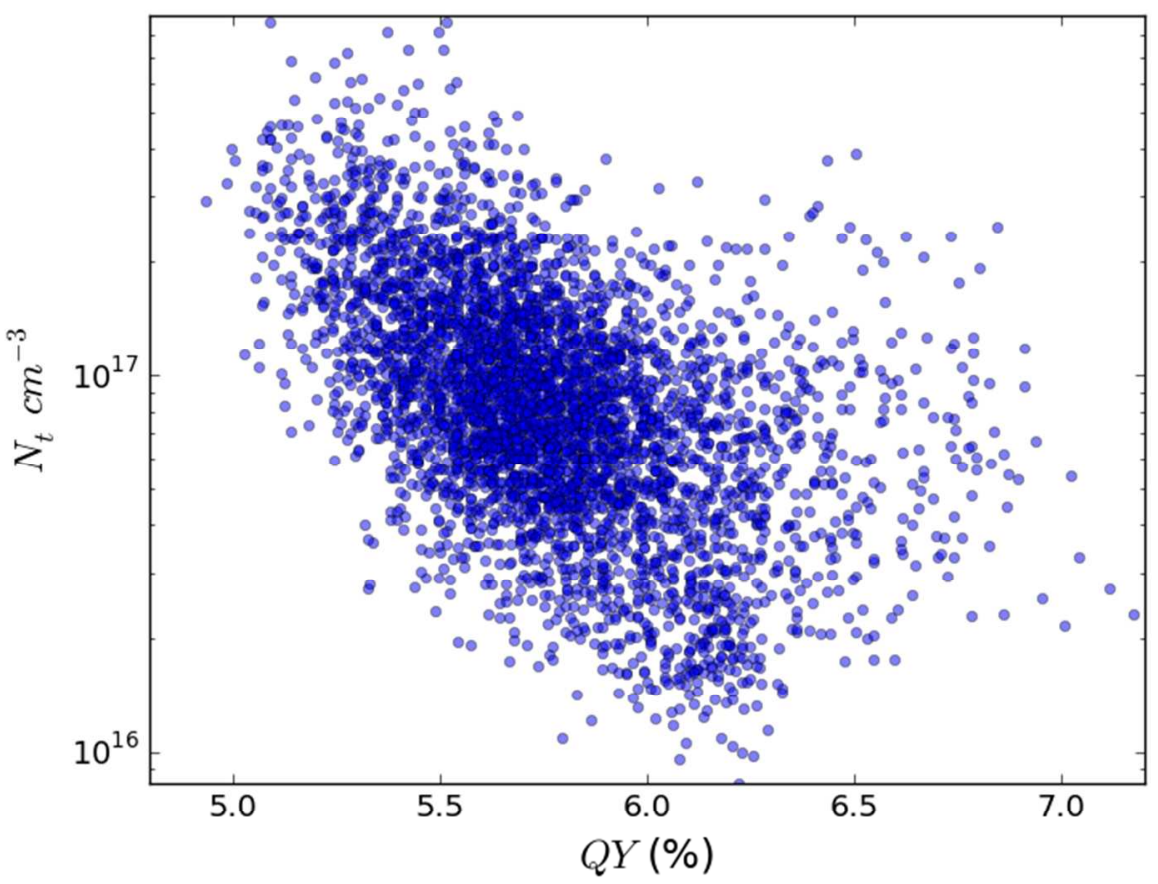

Figure S3. Pixel correlation plot between $N_{t}$ and $Q Y$ maps. 
(a)

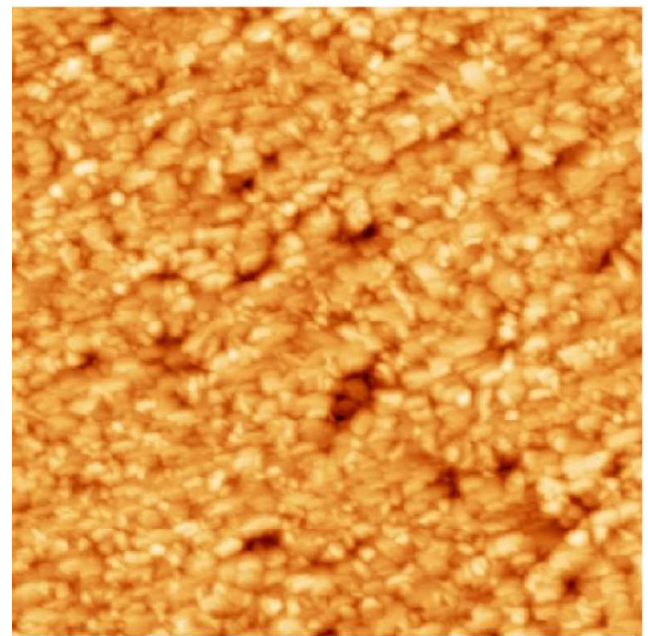

Figure S4. (a) AFM topography and (b) three-dimensional surface plot of a $\mathrm{MAPbI}_{3}$ thin film. The size of the AFM image is $5 \times 5 \mathrm{~m}^{2}$. (b)

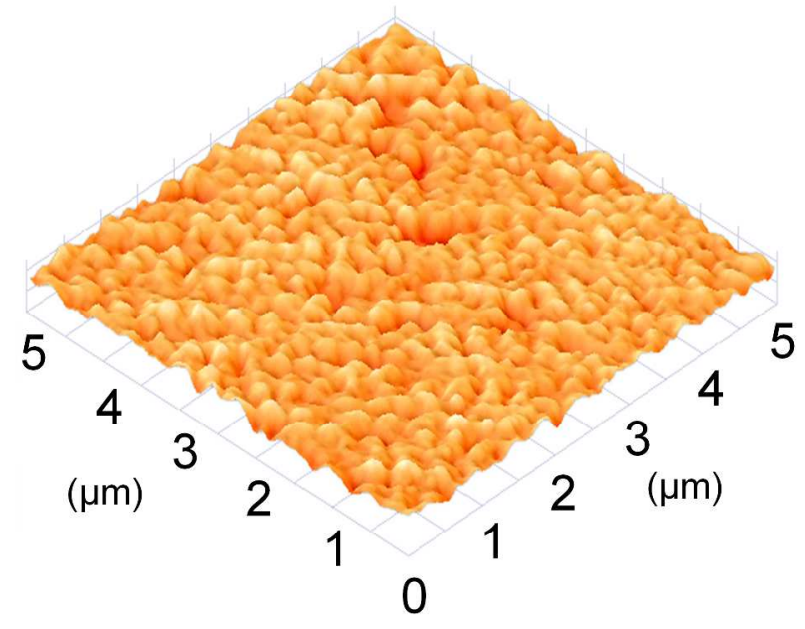

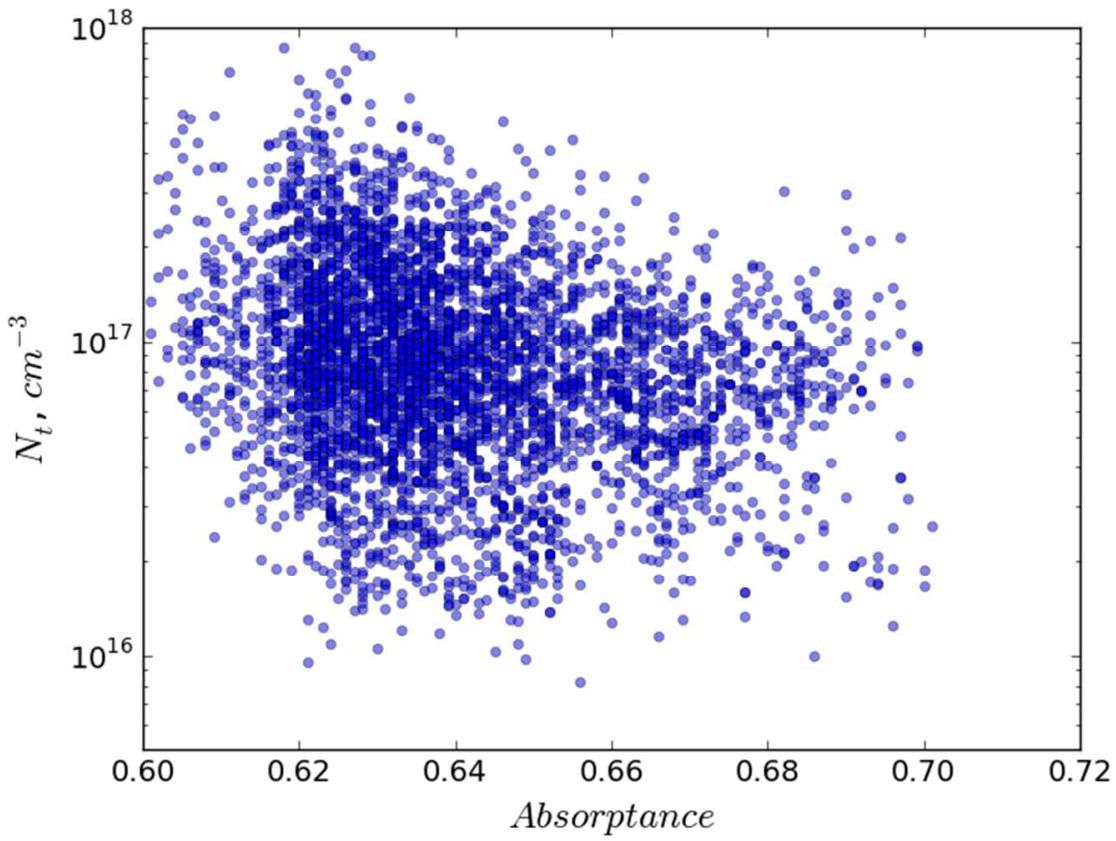

Figure S5. Pixel correlation plot between $N_{t}$ and $A$ (absorptance) maps. 


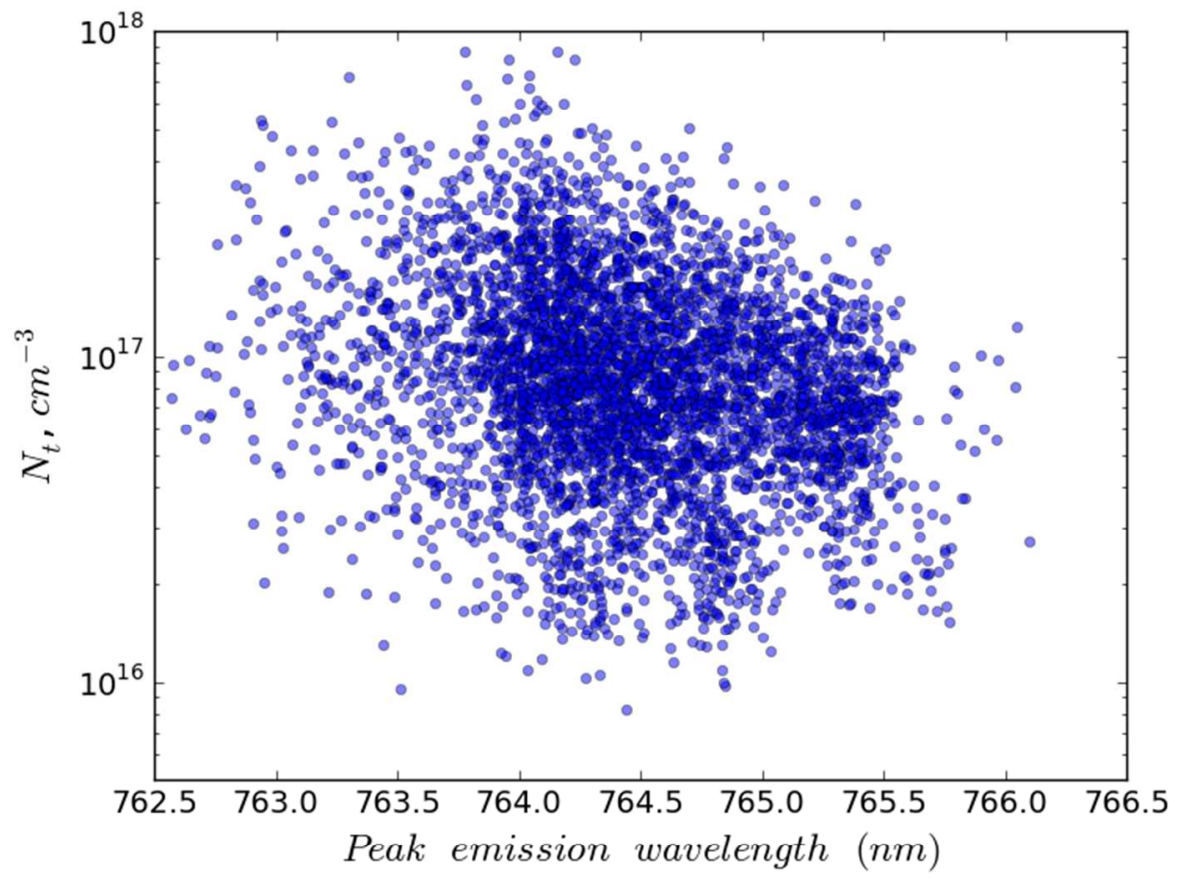

Figure S6. Pixel correlation plot between $N_{t}$ and peak emission wavelength maps.

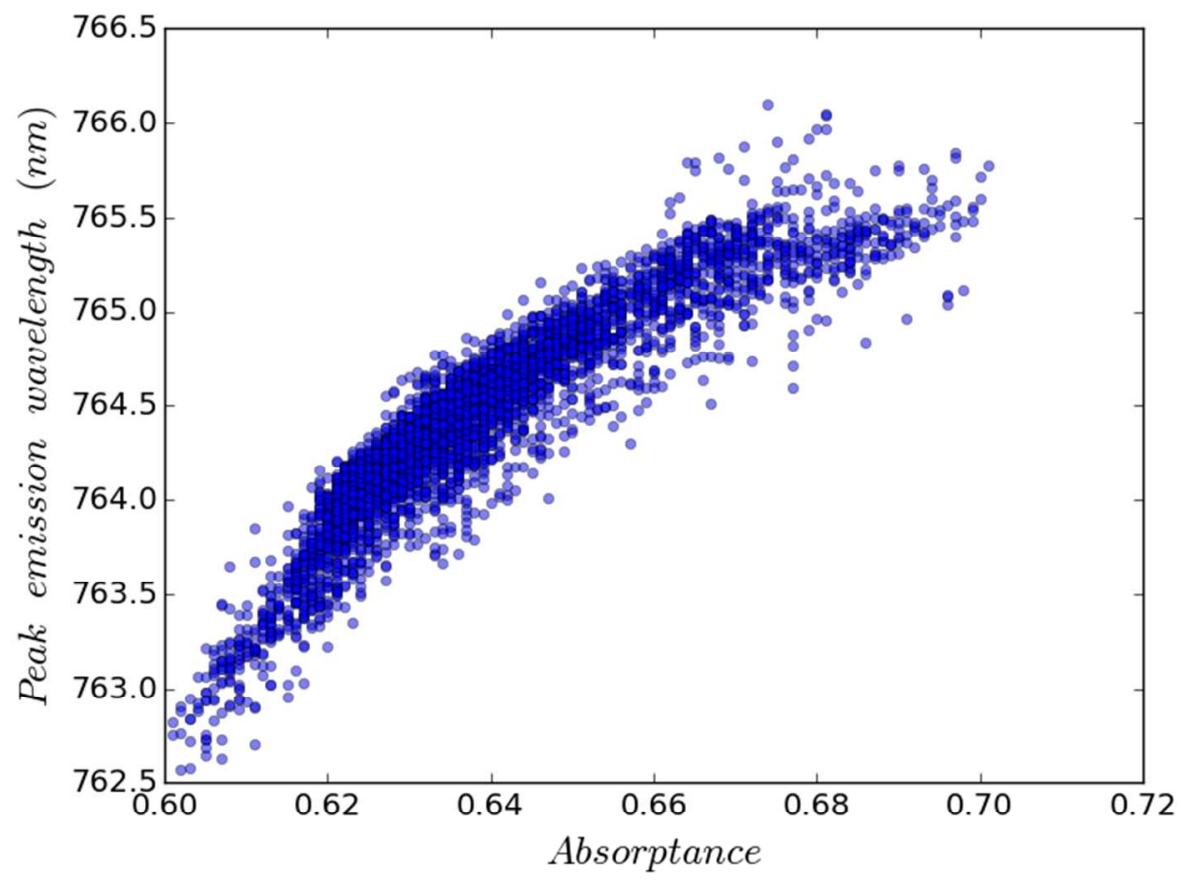

Figure S7. Pixel correlation plot between peak emission wavelength and $A$ maps. 


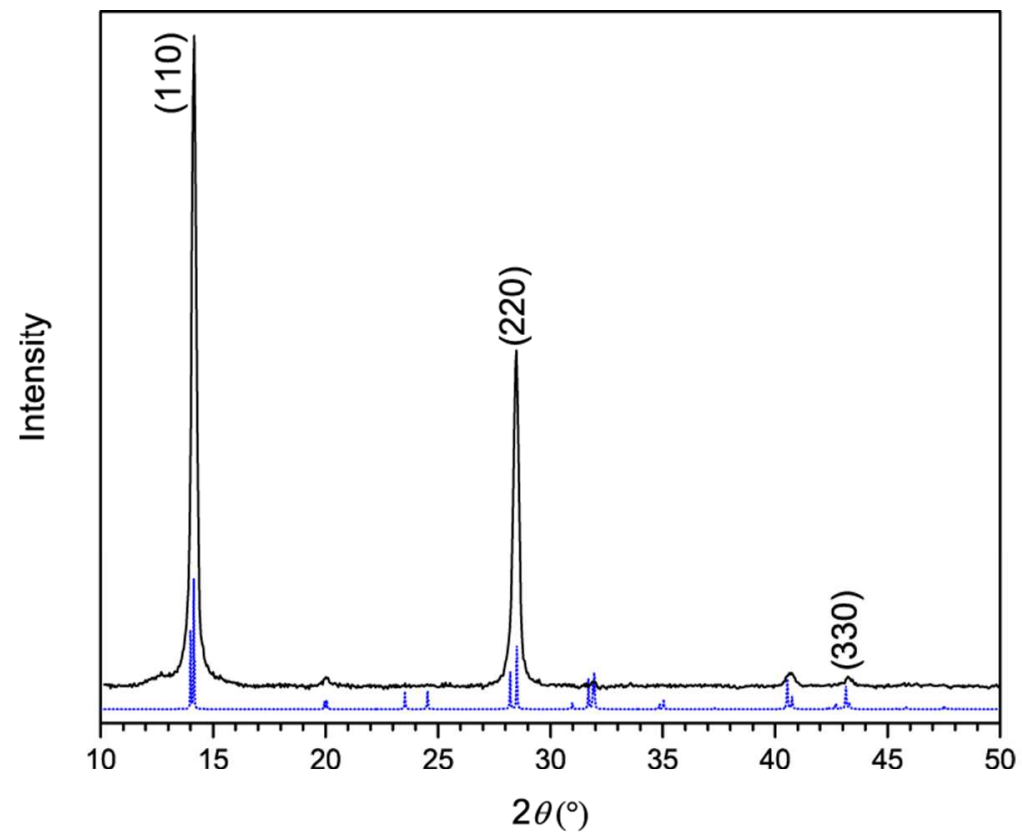

Figure S8. Representative $\mathrm{Cu}-\mathrm{K} \alpha$ powder-XRD pattern of solution-processed $\mathrm{CH}_{3} \mathrm{NH}_{3} \mathrm{PbI}_{3}$ thin film used for spatially resolved optical measurements (solid black line). Self-assembly results in preferred (110) orientation with the $c$-axis parallel to the substrate surface. The simulated pXRD pattern of tetragonal phase $\mathrm{CH}_{3} \mathrm{NH}_{3} \mathrm{PbI}_{3}$ (space group $I 4 \mathrm{~cm}$ ) is shown for reference (dotted blue line).

(1) Stranks, S. D.; Burlakov, V. M.; Leijtens, T.; Ball, J. M.; Goriely, A.; Snaith, H. J. Recombination Kinetics in Organic-Inorganic Perovskites: Excitons, Free Charge, and Subgap States. Phys. Rev. Appl. 2014, 2, 034007.

(2) Saba, M.; Cadelano, M.; Marongiu, D.; Chen, F.; Sarritzu, V.; Sestu, N.; Figus, C.; Aresti, M.; Piras, R.; Geddo Lehmann, A.; et al. Correlated Electron-Hole Plasma in Organometal Perovskites. Nat. Commun. 2014, 5, 5049. 